\title{
Maternal, antenatal and birth-associated determinants of neonatal iron stores
}

\author{
E.K. McCarthy ${ }^{1,2}$, L.C. Kenny ${ }^{2,3}$, J.O’B. Hourihane ${ }^{2,4}$, A.D. Irvine ${ }^{5,6,7}$, D.M. Murray ${ }^{2,4}$ and \\ M. Kiely ${ }^{1,2}$ \\ ${ }^{1}$ Cork Centre for Vitamin D and Nutrition Research, ${ }^{2}$ The Irish Centre for Fetal and Neonatal Translational Research \\ (INFANT), ${ }^{3}$ Department of Obstetrics and Gynaecology, and ${ }^{4}$ Department of Paediatrics and Child Health, University \\ College Cork, Republic of Ireland, ${ }^{5}$ Department of Clinical Medicine, Trinity College, ${ }^{6}$ Department of Paediatric \\ Dermatology, Our Lady's Children's Hospital and ${ }^{7}$ National Children's Research Centre, Dublin, Republic of Ireland
}

Serum ferritin concentrations measured in umbilical cord blood at birth are reflective of neonatal iron stores ${ }^{(1)}$. Iron stores in the neonate can track through to early childhood; those with the lowest ferritin concentrations at birth continue to have significantly lower ferritin concentrations up to two years of age ${ }^{(2)}$. Moreover, low iron stores at birth have been associated with adverse neurodevelopmental outcomes later in childhood ${ }^{(3)}$. The aim of this study was to identify maternal, antenatal and birth-associated determinants of serum ferritin concentrations at birth and to explore associations with iron status at two years of age.

Data were collected prospectively in the maternal-infant dyad Cork BASELINE Birth Cohort Study from 15 weeks' gestation throughout early childhood ${ }^{(4)}$. Only participants with a complete maternal-infant dataset with matched blood samples collected from infants in the umbilical cord at birth and at the 24-month assessment were included $(n=413)$. Serum ferritin concentrations were measured at birth and at two years, with haemoglobin concentrations and mean corpuscular volume also measured at two years. Regression models were developed to identify independent determinants of cord serum ferritin concentrations.

Mean (SD) cord serum ferritin concentrations were 236.5 (136.1) $\mu \mathrm{g} / \mathrm{l}$. Delivery by Caesarean section (adj. estimate (95\% confidence interval $(\mathrm{CI})) ;-41.8(-72 \cdot 6,-10 \cdot 9))$ and maternal obesity $\left(\mathrm{BMI} \geq 30 \mathrm{~kg} / \mathrm{m}^{2}\right)$ at 15 weeks' gestation $(-66 \cdot 4(-106 \cdot 9,-25.9)$, all $P<0.05$ ) were inversely associated with cord ferritin concentrations. Low iron stores (ferritin $<76 \mu \mathrm{g} / \mathrm{l}^{(3)}$ ) at birth were observed in $8 \%(n=33)$ of infants. Maternal smoking at 15 weeks' gestation (adj. odds ratio (95\% CI); $2.9(1.2,7 \cdot 0))$ and being born small-for-gestational age $(3.4(1.3,8.9)$, all $P<0.05)$ were associated with an increased risk of low iron stores at birth. Maternal anaemia (haemoglobin $<110 \mathrm{~g} / 1$ at 20 weeks' gestation) was not a significant determinant of cord ferritin concentrations. Cord ferritin concentrations were positively associated with ferritin concentrations at two years (adj. estimate (95\% CI); 0.03 ( 0.02 , $0.04), P<0.001)$.

Delivery by Caesarean section, being born small-for-gestational age and an unhealthy maternal lifestyle, characterised by smoking and obesity in pregnancy, were all associated with significantly lower serum ferritin concentrations at birth, which tracked through to two years of age. This study has highlighted the importance of overall maternal health during pregnancy for neonatal iron status. As some of these determinants are modifiable lifestyle factors, public health policies targeting women of child-bearing age to improve nutrition and health outcomes should be prioritised for the health of the next generation.

Research supported by the National Children's Research Centre and Danone Nutricia Early Life Nutrition.

1. Siimes MA, Addiego JE Jr., Dallman PR. (1974) Blood 43(4): 581-90

2. Hay G, Refsum H, Whitelaw A, et al. (2007) Am J Clin Nutr 86(1): 64-73.

3. Tamura T, Goldenberg RL, Hou J, et al. (2002) J Pediatr 140(2): 165-70.

4. O’Donovan SM, Murray DM, Hourihane JO, et al. (2015) Int J Epidemiol 44(3):764-75. 